\title{
How Many Turing Degrees are There?
}

\author{
Randall Dougherty and Alexander S. Kechris
}

\begin{abstract}
A Borel equivalence relation on a Polish space is countable if all of its equivalence classes are countable. Standard examples of countable Borel equivalence relations (on the space of subsets of the integers) that occur in recursion theory are: recursive isomorphism, Turing equivalence, arithmetic equivalence, etc. There is a canonical hierarchy of complexity of countable Borel equivalence relations imposed by the notion of Borel reducibility. We will survey results and conjectures concerning the problem of identifying the place in this hierarchy of these equivalence relations from recursion theory and also discuss some of their implications.
\end{abstract}

The obvious answer to the question of the title is: continuum many. There is however a different way of looking at this question, which leads to some very interesting open problems in the interface of recursion theory and descriptive set theory. Our goal in this paper is to explain the context in which this and related problems can be formulated, i.e., the theory of Borel equivalence relations, and survey some of the progress to date.

\section{Formulation of the problem}

We denote by $\equiv_{T}$ the Turing equivalence relation on $\mathcal{P}(\mathbb{N})=\{X: X \subseteq$ $\mathbb{N}\}$, which we identify with $2^{\mathbb{N}}$, viewing sets as characteristic functions. (We use the standard set-theoretic convention that $n=\{0,1, \ldots, n-1\}$ for all natural numbers $n$.) Then $\equiv_{T}$ is a Borel (in fact $\Sigma_{3}^{0}$ ) equivalence relation on $2^{\mathbb{N}}$. We denote by $\mathcal{D}$ the quotient space $2^{\mathbb{N}} /\left(\equiv_{T}\right)$, i.e., the set of Turing degrees.

Now consider general Borel equivalence relations on $2^{\mathbb{N}}$ or even arbitrary Polish (separable completely metrizable) spaces. We measure their complexity by studying the following partial (pre)order of Borel reducibility: if $E, F$ are Borel equivalence relations on $X, Y$ respectively, then a Borel reduction of $E$ into $F$ is a Borel map $f: X \rightarrow Y$ such that

$$
x E y \Longleftrightarrow f(x) F f(y)
$$

If such an $f$ exists we say that $E$ is Borel reducible to $F$ and denote this by

$$
E \leq_{B} F .
$$

Let also

$$
E \sim_{B} F \Longleftrightarrow E \leq_{B} F \& F \leq_{B} E
$$

1991 Mathematics Subject Classification. Primary 03D30, 03E15; Secondary 04A15, 54H05. The first author was partially supported by NSF Grant DMS 9158092.

The second author was partially supported by NSF Grant DMS 9619880.

(c) 2000 American Mathematical Society 
(this defines the concept of bi-reducibility) and

$$
E<_{B} F \Longleftrightarrow E \leq_{B} F \& F \leq_{B} E .
$$

Let us say that a function $f_{*}: X / E \rightarrow Y / F$ is Borel if it has a Borel lifting, i.e., there is a Borel function $f: X \rightarrow Y$ such that $f_{*}\left([x]_{E}\right)=[f(x)]_{F}$ for all $x \in X$. Then it is clear that $E \leq_{B} F$ is equivalent to the assertion that there is a Borel injection from $X / E$ into $Y / F$, which we express by saying that the Borel cardinality, $|E|_{B}$, of $E$ is less than or equal to to that of $F$; in symbols,

$$
|E|_{B} \leq|F|_{B} \Longleftrightarrow E \leq_{B} F \text {. }
$$

Then define

$$
|E|_{B}=|F|_{B} \Longleftrightarrow E \sim_{B} F
$$

i.e., $X / E, Y / F$ have the same Borel cardinality, and

$$
|E|_{B}<|F|_{B} \Longleftrightarrow E<_{B} F
$$

i.e., $X / E$ has (strictly) smaller Borel cardinality then $Y / F$.

We are now ready to formulate our problem as follows, where, by abusing notation, we write below $|\mathcal{D}|_{B}$ instead of $\left|\equiv_{T}\right|_{B}$ and call this the Borel cardinality of $\mathcal{D}$, instead of $\equiv_{T}$ :

Question: What is the Borel cardinality, $|\mathcal{D}|_{B}$, of the set of Turing degrees $\mathcal{D}$ ?

If we denote the classical (Cantor) cardinality of $\mathcal{D}$ by $|\mathcal{D}|$, then we have $|\mathcal{D}|=$ $|\mathbb{R}|$. However, it is not hard to see that the Borel cardinality of $\mathcal{D}$ is bigger than that of the continuum. Let $=_{X}$ be the identity relation on the Polish space $X$. So $|=\mathbb{R}|_{B}$ is the Borel cardinality which naturally represents the classical cardinality of the continuum.

Fact. $\left(\equiv_{T}\right)>_{B}\left(=_{\mathbb{R}}\right)$.

Proof. It is standard that there is a perfect set of pairwise Turing incomparable subsets of $\mathbb{N}$, so $\left(=_{\mathbb{R}}\right) \leq_{B}\left(\equiv_{T^{\prime}}\right)$. If on the other hand $f: 2^{\mathbb{N}} \rightarrow \mathbb{R}$ is Borel and Turing-invariant, i.e., $x \equiv_{T} y \Longrightarrow f(x)=f(y)$, then for each Borel set $A \subseteq \mathbb{R}, f^{-1}(A)$ is a Turing-invariant Borel subset of $2^{\mathbb{N}}$, so it has measure 0 or 1. It follows that, for each $n$, the $n$th digit in the decimal expansion of $f(x)$ is fixed on a set of measure 1. So there is a Turing-invariant Borel set of measure 1 on which $f$ is constant, therefore $f$ cannot be a reduction of $\equiv_{T}$ into $=_{\mathbb{R}}$. Thus $\left(\equiv_{T}\right) \underline{\Sigma}_{B}\left(=_{\mathbb{R}}\right)$.

We now have our question but it is not clear yet what kind of answer we should expect. In what sense can we hope to compute $|\mathcal{D}|_{B}$ ? To understand this, we have to dig a little deeper into the theory of Borel equivalence relations.

For our purposes, a crucial property of the Turing equivalence relation is that it has countable equivalence classes. In general, we call a Borel equivalence relation countable if every one of its classes is countable. We will next review some basic facts of the theory of countable Borel equivalence relations, for which we refer the reader to the papers Kechris [K2], Dougherty-Jackson-Kechris [DJK], JacksonKechris-Louveau [JKL], Kechris [K1], and Adams-Kechris [AK].

(i) (Feldman-Moore $[\mathbf{F M}]$ ) Every countable Borel equivalence relation is generated by a Borel action of a countable group. 
More precisely, given a countable Borel equivalence $E$ on a Polish space $X$, there is a countable group $G$ and a Borel action $(g, x) \mapsto g \cdot x$ of $G$ on $X$ such that, if $E_{G}^{X}$ is defined by

then $E=E_{G}^{X}$.

$$
x E_{G}^{X} y \Longleftrightarrow \exists g \in G(g \cdot x=y),
$$

In particular, $\equiv_{T}$ is given by a Borel action of a countable group on $2^{\mathbb{N}}$. It seems like an interesting, but somewhat vague, question to find out whether one can obtain such a representation that has some recursion theoretic significance.

REMARK 1.1. Using the Feldman-Moore theorem and related facts, within a Schröder-Bernstein argument, one can show that, for countable Borel equivalence relations $E$ and $F, E \sim_{B} F$ is equivalent to the existence of a Borel bijection of $X / E$ with $Y / F$.

(ii) There is a universal countable Borel equivalence relation, in the sense of $\leq_{B}$.

That is, there is a countable Borel equivalence relation $E$ such that, for any countable Borel equivalence relation $F$, we have $F \leq_{B} E$. This $E$ is clearly unique, up to $\sim_{B}$, and denoted by $E_{\infty}$.

An example of a universal countable Borel equivalence is given by the orbit equivalence relation of the shift action of $F_{2}$, the free group on two generators, on $2^{F_{2}}$ given by

$$
g \cdot x(h)=x\left(g^{-1} h\right), \quad g, h \in F_{2}, \quad x \in 2^{F_{2}} .
$$

(iii) There is a smallest, in the sense of $\leq_{B}$, countable Borel equivalence relation on uncountable Polish spaces, namely $=_{\mathbb{k}}$.

So for every countable Borel equivalence relation $E$ on an uncountable Polish space, we have $\left(=_{\mathbb{R}}\right) \leq_{B} E$. If $\left(=_{\mathbb{R}}\right) \sim_{B} E$, we say that $E$ is smooth. For example, $\equiv_{T}$ is not smooth. Another example of a non-smooth countable Borel equivalence is the following one, defined on $2^{\mathbb{N}}$ :

$$
x E_{0} y \Longleftrightarrow \exists n \forall m \geq n(x(m)=y(m)) .
$$

This turns out to be the smallest, in the sense of $\leq_{B}$, non-smooth countable Borel equivalence relation. This is a particular instance of the general Glimm-Effros Dichotomy proved in Harrington-Kechris-Louveau [HKL], but this special case can already be derived from Effros $[\mathbf{E}]$.

(iv) (Glimm-Effros Dichotomy) If $E$ is a countable Borel equivalence relation which is not smooth, then $E_{0} \leq_{B} E$.

(v) $E_{0}<_{B} E_{\infty}$.

Thus we have

$$
\left(==_{\mathbb{R}}\right)<_{B} E_{0}<_{B} E_{\infty}
$$

and every other countable Borel equivalence relation on an uncountable space is in the interval $\left(E_{0}, E_{\infty}\right)$.

(vi) (Adams-Kechris $[\mathbf{A K}]$ ) There are continuum many pairwise incomparable, under $\leq_{B}$, countable Borel equivalence relations.

We now have all the ingredients to formulate a precise conjecture, in response to the question about the Borel cardinality of $\mathcal{D}$. This was originally formulated (as 
a question) in Kechris [K2] and listed (as a conjecture) in Slaman's list of Questions in Recursion Theory, item 2.3, posted in http://math.berkeley.edu/ slaman/.

Conjecture: $\equiv_{T}$ is a universal countable Borel equivalence relation, i.e., $\left(\equiv_{T}\right) \sim_{B}$ $E_{\infty}$.

\section{Known results and implications}

There is some information already available about the complexity of $\equiv_{T}$.

THEOREM 2.1. (Slaman-Steel $[\mathbf{S S}]) E_{0}<_{B}\left(\equiv_{T}\right)$.

This has been strengthened in Kechris [K1] to show that $\equiv_{T}$ is not amenable and in Jackson-Kechris-Louveau $[\mathbf{J K L}]$ to show that $\equiv_{T}$ is not treeable, all indications that $\equiv_{T}$ is quite complex.

One of the intriguing implications of the conjecture that $\equiv_{T}$ is universal concerns the existence of unusual functions on the Turing degrees. Recall that we call a function $f: \mathcal{D}^{n} \rightarrow \mathcal{D}$ Borel if there is a Borel function $F:\left(2^{\mathbb{N}}\right)^{n} \rightarrow 2^{\mathbb{N}}$ such that

$$
f\left(\left[x_{1}\right]_{T}, \ldots,\left[x_{n}\right]_{T}\right)=\left[F\left(x_{1}, \ldots, x_{n}\right)\right]_{T}
$$

for all $x_{1}, \ldots, x_{n} \in 2^{\mathbb{N}}$, where $[x]_{T}$ is the Turing degree of $x \in 2^{\mathbb{N}}$. A pairing function on $\mathcal{D}$ is a bijection $\langle\rangle:, \mathcal{D}^{2} \rightarrow \mathcal{D}$.

Fact. If $\equiv_{T}$ is universal, then there is a Borel pairing function on $\mathcal{D}$.

Proof. If $E, F$ are Borel equivalence relations on $X, Y$ respectively, let $E \times F$ be the Borel equivalence relation on $X \times Y$ given by

$$
(x, y)(E \times F)\left(x^{\prime}, y^{\prime}\right) \Longleftrightarrow x E x^{\prime} \& y F y^{\prime} .
$$

Clearly $E_{\infty} \times E_{\infty} \geq_{B} E_{\infty}$, so, since $E_{\infty}$ is universal, $E_{\infty} \times E_{\infty} \sim_{B} E_{\infty}$. Hence, if $\left(\equiv_{T}\right) \sim_{B} E_{\infty}$, we have

$$
\left(\equiv_{T}\right) \times\left(\equiv_{T}\right) \sim_{B}\left(\equiv_{T}\right),
$$

which shows that there is a Borel pairing function on $\mathcal{D}$.

The well-known Martin Conjecture (or the 5th Victoria Delfino problem), see Kechris-Moschovakis, Eds. [KM] or Slaman's list, item 2.2, seeks to classify definable functions on $\mathcal{D}$, asymptotically, i.e., up to identification on a cone of degrees. One part of the conjecture asserts, in particular, that if a Borel $f: \mathcal{D} \rightarrow \mathcal{D}$ is not constant on a cone, then $f(d) \geq d$ on a cone. We can now easily see the following:

Fact. If $\equiv_{T}$ is universal, then Martin's Conjecture fails.

Proof. Fix $d_{0} \neq d_{1}$ in $\mathcal{D}$ and let $\langle$,$\rangle be a Borel pairing function on \mathcal{D}$. Let $f_{0}(d)=\left\langle d_{0}, d\right\rangle$ and $f_{1}(d)=\left\langle d_{1}, d\right\rangle$. Then $f_{i}: \mathcal{D} \rightarrow \mathcal{D}$ is Borel for $i=0,1$ and, if $A_{i}=\operatorname{rng}\left(f_{i}\right)$, then $A_{0} \cap A_{1}=\varnothing$. Since $\equiv_{T}$ is countable, one can show that the inverse of the pairing function $\langle$,$\rangle is also Borel, so the sets A_{i}$ are Borel.

Clearly $f_{0}$ and $f_{1}$ are injective, so they are not constant on a cone. Thus, if Martin's Conjecture were true, we would have that $f_{i}(d) \geq d$ on a cone for $i=0,1$. Then $A_{0}$ and $A_{1}$ would be cofinal in the Turing degrees, so, by Borel Determinacy, each would contain a cone, contradiction. 


\section{Some more questions and answers}

There are of course several other notions of equivalence and degree studied in recursion theory, and similar questions and conjecture can be considered for them too. We will concentrate here on one of the finest, recursive isomorphism, and one of the coarsest, arithmetic equivalence.

Let $S_{\infty}$ be the group of permutations of $\mathbb{N}$, and let $S_{r}$ be the subgroup consisting of all recursive permutations. We let $\equiv_{r}$ denote recursive isomorphism for subsets of $\mathbb{N}$. Via our identification of $\mathcal{P}(\mathbb{N})$ with $2^{\mathbb{N}}$, we have for $x, y \in 2^{\mathbb{N}}$ :

$$
x \equiv_{r} y \Longleftrightarrow \exists \pi \in S_{r}(x \circ \pi=y) .
$$

For any $n \in\{2,3,4, \ldots\} \cup\{\mathbb{N}\}$ we also define recursive isomorphism on $n^{\mathbb{N}}$ by

$$
x \equiv_{r}^{n} y \Longleftrightarrow \exists \pi \in S_{r}(x \circ \pi=y),
$$

so that $\left(\equiv_{r}^{2}\right)=\left(\equiv_{r}\right)$.

It is well-known that $\left(\equiv_{T}\right) \leq_{B}\left(\equiv_{T}\right)$, because $x \equiv_{T} y \Longleftrightarrow x^{\prime} \equiv_{r} y^{\prime}$, where $x^{\prime}$ is the Turing jump of $x$. Hence, if $\equiv_{T}$ is universal, then $\equiv_{r}$ is universal; and proving that $\equiv_{r}$ is universal could be viewed as providing additional evidence that $\equiv_{T}$ is universal.

Finally, we denote by $\equiv_{A}$ the notion of arithmetic equivalence on $2^{\mathbb{N}}$. So $\left(\equiv_{r}\right) \subseteq\left(\equiv_{T}\right) \subseteq\left(\equiv_{A}\right)$.

Again, one can conjecture that $\equiv_{r}$ and $\equiv_{A}$ are universal. Here, though, we have some answers.

Theorem 3.1. (Slaman-Steel, unpublished). Arithmetic equivalence, $\equiv_{A}$, is universal, i.e., $\left(\equiv_{A}\right) \sim_{B} E_{\infty}$.

So arithmetical equivalence has a Borel pairing function, and the arithmetical analogue of Martin's Conjecture fails.

The problem for recursive equivalence is still open, but there has been a lot of progress.

THEOREM 3.2. (Dougherty-Kechris [DK]). Recursive isomorphism on $\mathbb{N}^{\mathbb{N}}$ is universal, i.e., $\left(\equiv_{r}^{\mathbb{N}}\right) \sim_{B} E_{\infty}$.

This was very recently improved to

Theorem 3.3. (Andretta-Camerlo-Hjorth $[\mathbf{A C H}]$ ). Recursive isomorphism on $5^{\mathbb{N}}$ is universal, i.e., $\left(\equiv_{r}^{5}\right) \sim_{B} E_{\infty}$.

However, it is not yet clear how to reduce 5 to 2 .

Actually, Theorems 3.2 and 3.3 are much more general. In each case, one actually shows that there is a fixed subgroup $S_{0}$ consisting of primitive recursive (in fact much simpler) permutations such that the result is true if $S_{r}$ is replaced by any countable group $S$ with $S_{0} \subseteq S \subseteq S_{\infty}$.

There is one last problem related to Theorem 3.2, that has further interesting implications.

First recall that an action of a group $G$ on a set $X$ is called free if $g \cdot x \neq$ $x$ for any $x \in X$ and $g \neq 1_{G}$. Also recall from $\S 2$ that every countable Borel equivalence relation is induced by a Borel action of a countable group $G$. From considerations in ergodic theory, it turns out that it is not always possible to find a free such action that induces it; see Adams $[\mathbf{A}]$. It has been observed though that every known example of a countable Borel equivalence relation $E$, which cannot 
be induced by a free Borel action of a countable group, admits an invariant Borel probability measure (measure for short). (A measure is invariant for $E$ if it is invariant for any Borel action of a countable group that generates it.) It has in fact been conjectured that this is always the case. In other words, a countable Borel equivalence relation which does not admit an invariant measure can be induced by a free Borel action of a countable group.

By using the arguments in $\S 2$ of Dougherty-Jackson-Kechris [DJK] and a theorem of Nadkarni $[\mathbf{N}]$, it can be seen that this last assertion is equivalent to the following:

$(\dagger)$ There is a universal countable Borel equivalence relation, which is induced by a free Borel action of a countable group.

We return now to Theorem 3.2. We have that $\equiv_{r}^{\mathbb{N}}$ is induced by the following Borel action of $S_{r}$ on $\mathbb{N}^{\mathbb{N}}$ :

$$
\pi \cdot x=x \circ \pi^{-1} \text {. }
$$

This action is not free, but its restriction to

$$
[\mathbb{N}]^{\mathbb{N}}=\left\{x \in \mathbb{N}^{\mathbb{N}}: x \text { is one-to-one }\right\}
$$

is. It is natural to conjecture that Theorem 3.2 can be strengthened to the statement that $\left(\equiv_{r}\right) \uparrow[\mathbb{N}]^{\mathbb{N}}$ is universal. If this turns out to be the case, this will also prove $(\dagger)$.

\section{Some proofs}

We will give here our proof of Theorem 3.2 (and a related result). This comes from the unpublished Dougherty-Kechris [DK]. Although Theorem 3.2 has now been superseded by Theorem 3.3, our proof uses different methods and may find other applications in the future.

As we indicated in $\S 3$, one has in fact a stronger result. For any subgroup $S$ of $S_{\infty}$, and any $X$, let for $x, y \in X^{\mathbb{N}}$ :

$$
x \equiv \stackrel{X}{X} y \Longleftrightarrow \exists \pi \in S(x \circ \pi=y) .
$$

So $\left(\equiv_{r}^{\mathbb{N}}\right)=\left(\equiv_{S_{r}}^{\mathbb{N}}\right)$. We call $S$ primitive recursive if $S=\left\{g_{n}: n \in \mathbb{N}\right\}$, with $g(n, m)=g_{n}(m)$ primitive recursive. We now have:

THEOREM 4.1. There is a primitive recursive countable group $S_{0} \subseteq S_{\infty}$ such that for any countable group $S$ with $S_{0} \subseteq S \subseteq S_{\infty}$, we have that $\equiv_{S}^{\mathbb{N}}$ is a universal countable Borel equivalence relation. In particular this is true for $\equiv_{r}^{\mathbb{N}}$.

Proof. To explain the basic idea, consider a countable infinite group $H$ and fix a one-to-one enumeration $H=\left\{h_{n}: n \in \mathbb{N}\right\}$ of it. Then any $h_{a} \in H$ corresponds to a permutation $\tilde{a} \in S_{\infty}$ given by $h_{\tilde{a}(n)}=h_{n} h_{a}$ (the right regular representation). Fix also a bijection $\langle\rangle:, \mathbb{N}^{2} \rightarrow \mathbb{N}$ and let $\pi_{a} \in S_{\infty}$ be defined by

$$
\pi_{a}(\langle n, m\rangle)=\langle\tilde{a}(n), m\rangle .
$$

Now given an action $(h, x) \mapsto h \cdot x$ of $H$ into a space of the form $X^{\mathbb{N}}$ and the corresponding equivalence relation $E_{H}$, define the function $f: X^{\mathbb{N}} \rightarrow X^{\mathbb{N}}$ by

$$
f(x)(\langle n, m\rangle)=\left(h_{n} \cdot x\right)(m) .
$$


Then we have

$$
\begin{aligned}
f\left(h_{a} \cdot x\right)(\langle n, m\rangle) & =\left(h_{n} \cdot\left(h_{a} \cdot x\right)\right)(m) \\
& =\left(h_{\tilde{a}(n)} \cdot x\right)(m) \\
& =f(x)(\langle\tilde{a}(n), m\rangle) \\
& =\left(f(x) \circ \pi_{a}\right)(\langle n, m\rangle) ;
\end{aligned}
$$

hence, $f\left(h_{a} \cdot x\right)=f(x) \circ \pi_{a}$. It follows that if $H_{0}=\left\{\pi_{a}: a \in \mathbb{N}\right\}$ (a countable subgroup of $\left.S_{\infty}\right)$, then

$$
x E_{H} y \Longleftrightarrow f(x) \equiv_{H_{0}}^{X} f(y) .
$$

Unfortunately, if $S_{\infty} \supseteq H^{\prime} \supseteq H_{0}, H^{\prime}$ a countable group, then we cannot, in general, replace $H_{0}$ by $H^{\prime}$ in $\left(^{*}\right)$ since it could be that $f(x) \equiv_{H^{\prime}}^{X} f(y)$ via some $\pi \in H^{\prime} \backslash H_{0}$. After appropriately choosing $H, X$, and the action of $H$ on $X^{\mathbb{N}}$ (so that at least $E_{H}$ is universal), we will modify $f(x)$ to $f^{*}(x) \in\left(X^{*}\right)^{\mathbb{N}}$, for some $X^{*}$, by encoding in it some further information, so that even if $f(x) \equiv_{H^{\prime}}^{X^{*}} f(y)$ via some $\pi \in H^{\prime} \backslash H_{0}$ we can still conclude that $x E_{H} y$. In particular, although the $X$ we will start with will be finite, this encoding will require $X^{*}$ to be infinite. Moreover, we will be forced to restrict the $x$ 's to some subset of $X^{\mathbb{N}}$, say $Y \subseteq X^{\mathbb{N}}$, so we will also need to make sure that $E_{H}\lceil Y$ is universal.

We will now implement this idea. We fix some notation first:

For any $X$ and countable group $G$, we have the shift action of $G$ on $X^{G}$ given by

$$
g \cdot x(h)=x\left(g^{-1} h\right)
$$

This induces for any subgroup $H \subseteq G$ an action of $H$ on $X^{G}$ and we denote the corresponding equivalence relation by $E\left(H, X^{G}\right)$. If $G$ is infinite, fixing a one-to-one enumeration of $G$, we can view this as an action of $H$ on $X^{\mathbb{N}}$.

Now fix a one-to-one enumeration $\left\{g_{n}: n \in \mathbb{N}\right\}$ of the free group $F_{2}$ on two generators, with $g_{0}=1$ where 1 is the identity element of $F_{2}$. Define $\tilde{a}$ and $\pi_{a}$ as above by the formulas $g_{\tilde{a}(n)}=g_{n} g_{a}$ and $\pi_{a}(\langle n, m\rangle)=\langle\tilde{a}(n), m\rangle$, and let

$$
S_{0}=\left\{\pi_{a}: a \in \mathbb{N}\right\}
$$

If $\left\{g_{n}: n \in \mathbb{N}\right\}$ and $\langle$,$\rangle are chosen appropriately, then S_{0}$ is primitive recursive. Fix also any countable group $S$ such that $S_{\infty} \supseteq S \supseteq S_{0}$; we will show that $\equiv_{S}^{\mathbb{N}}$ is universal. Say $S=\left\{\rho_{i}: i \in \mathbb{N}\right\}$.

We call $i \in \mathbb{N}$ bad if

(i) $\forall n \forall m \exists n^{\prime}\left(\rho_{i}(\langle n, m\rangle)=\left\langle n^{\prime}, m\right\rangle\right)$; and

(ii) if $\rho_{i}(\langle 0, m\rangle)=\left\langle n_{m}, m\right\rangle$ for all $m$, then $n_{m} \rightarrow \infty$ as $m \rightarrow \infty$.

We can now easily define $n_{j}^{(i)}, m_{j}^{(i)} \in \mathbb{N}$ for $i, j \in \mathbb{N}$ such that:

(a) $0<n_{j}^{(i)}<n_{j+1}^{(i)}$ and $0<m_{j}^{(i)}<m_{j+1}^{(i)}$;

(b) $(i, j) \neq\left(i^{\prime}, j^{\prime}\right) \Longrightarrow m_{j}^{(i)} \neq m_{j^{\prime}}^{\left(i^{\prime}\right)}$;

(c) if $i$ is bad, then $n_{m_{j}^{(i)}}=n_{j}^{(i)}$.

Also, for the free group $F_{k}$ with $k$ generators and $g \in F_{k}, m \in \mathbb{N}$, let $B_{k}(g, m)$ be the ball of radius $m$ around $g$ in the tree of $F_{k}$; i.e., $B_{k}(g, m)$ is the set of all products $g h$ where $h$ is a word in $F_{k}$ of length at most $m$. 
Now consider the shift action of $F_{2}$ on $9^{F_{3}}$ ( 9 is a large enough number here) and the Borel set $A \subseteq 9^{F_{3}}$ defined by

$$
\begin{gathered}
y \in A \Longleftrightarrow \forall i \forall j\left[\left[( g _ { n _ { j } ^ { ( i ) } } \cdot y ) \left\lceilB_{3}\left(1, m_{j}^{(i)}\right)=\left(g_{n_{j+1}^{(i)}} \cdot y\right)\left\lceil B_{3}\left(1, m_{j}^{(i)}\right)\right] \Longrightarrow\right.\right.\right. \\
\left.g_{n_{j}^{(i)}} \cdot y=g_{n_{j+1}^{(i)}} \cdot y\right],
\end{gathered}
$$

where 1 is the identity element of $F_{3}$.

Lemma 4.2. $E\left(F_{2}, 9^{F_{3}}\right)\left\lceil A \leq_{B}\left(\equiv_{S}^{\mathbb{N}}\right)\right.$.

Proof. Fix an injection $c$ from the countable set $\bigcup_{m} 9^{B_{3}(1, m)}$ to $\mathbb{N}$. Now define $f^{*}: A \rightarrow \mathbb{N}^{\mathbb{N}}$ by $f^{*}(x)=x^{*}$, where $x^{*}(\langle n, m\rangle)=c\left(\left(g_{n} \cdot x\right)\left\lceil B_{3}(1, m)\right)\right.$. Thus $x^{*}(\langle n, m\rangle)$ encodes the values of $g_{n} \cdot x$ at the ball of radius $m$ around $1 \in F_{3}$. In particular, $x^{*}(\langle n, m\rangle)$ encodes (i.e., uniquely determines) $m$ as well. (If we were to take $f(x)$ as in the intuitive explanation in the beginning of this proof, then $f(x)(\langle n, m\rangle)$ would be just $g_{n} \cdot x\left(p_{m}\right)$, where $\left\{p_{m}: m \in \mathbb{N}\right\}$ is a one-to-one enumeration of $F_{3}$.)

We claim that

$$
x E\left(F_{2}, 9^{F_{3}}\right) y \Longleftrightarrow x^{*} \equiv_{S}^{\mathbb{N}} y^{*},
$$

which completes the proof.

$\Rightarrow$ : Clearly $y=g_{a} \cdot x \Longrightarrow y^{*}=x^{*} \circ \pi_{a}$.

$\Leftarrow$ : Say now $\pi \in S$ is such that $y^{*}=x^{*} \circ \pi$, i.e., $y^{*}(\langle n, m\rangle)=x^{*}(\pi(\langle n, m\rangle))$. Since $x^{*}(\langle n, m\rangle)$ encodes $m$, it follows that there is a function $\pi^{\prime}: \mathbb{N} \rightarrow \mathbb{N}$ such that $\pi(\langle n, m\rangle)=\left\langle\pi^{\prime}(\langle n, m\rangle), m\right\rangle$ for all $n$ and $m$; that is, the second coordinate is left fixed by $\pi$. (Note that all $\pi_{a}$ have this property, of course. By our encoding we have forced any $\pi$ as above to have it as well.)

We now have two cases:

(I) $\pi^{\prime}(0, m)$ does not tend to $\infty$ as $m \rightarrow \infty$. So there must exist a number $\ell$ such that, for infinitely many $m, \pi^{\prime}(0, m)=\ell$. For any such $m$, we have $y^{*}(\langle 0, m\rangle)=$ $x^{*}(\langle\ell, m\rangle)$, i.e., $y\left\lceil B_{3}(1, m)=\left(g_{\ell} \cdot x\right)\left\lceil B_{3}(1, m)\right.\right.$; since there are arbitrarily large such $m$, it follows that $y=g_{\ell} \cdot x$, so $x E\left(F_{2}, 9^{F_{3}}\right) y$.

(II) $\pi^{\prime}(0, m) \rightarrow \infty$ as $m \rightarrow \infty$. So if $\pi=\rho_{i}$, then $i$ is bad. For any $j$, we have $y^{*}\left(\left\langle 0, m_{j}^{(i)}\right\rangle\right)=x^{*}\left(\left\langle n_{j}^{(i)}, m_{j}^{(i)}\right\rangle\right)$, i.e., $y\left\lceil B_{3}\left(1, m_{j}^{(i)}\right)=\left(g_{n_{j}^{(i)}} \cdot x\right)\left\lceil B_{3}\left(1, m_{j}^{(i)}\right)\right.\right.$; but we also have $y\left\lceil B_{3}\left(1, m_{j+1}^{(i)}\right)=\left(g_{n_{j+1}^{(i)}} \cdot x\right)\left\lceil B_{3}\left(1, m_{j+1}^{(i)}\right)\right.\right.$, and $m_{j}^{(i)}<m_{j+1}^{(i)}$, so we get $\left(g_{n_{j}^{(i)}} \cdot x\right)\left\lceil B_{3}\left(1, m_{j}^{(i)}\right)=\left(g_{n_{j+1}^{(i)}} \cdot x\right)\left\lceil B_{3}\left(1, m_{j}^{(i)}\right)\right.\right.$. So, since $x \in A$, we have $g_{n_{j}^{(i)}} \cdot x=g_{n_{j+1}^{(i)}} \cdot x$ for all $j$, i.e., $g_{n_{0}^{(i)}} \cdot x=g_{n_{1}^{(i)}} \cdot x=g_{n_{2}^{(i)}}: x=\ldots$. It follows that $y\left|B_{3}\left(1, m_{j}^{(i)}\right)=\left(g_{n_{0}^{(i)}} \cdot x\right)\right| B_{3}\left(1, m_{j}^{(i)}\right)$ for all $j$; since $m_{j}^{(i)} \rightarrow \infty$ as $j \rightarrow \infty$, we have $y=g_{n_{0}^{(i)}} \cdot x$, so $x E\left(F_{2}, 9^{F_{3}}\right) y$ again.

It remains to show that $E\left(F_{2}, 9^{F_{3}}\right)\lceil A$ is universal. For that we will show that

$$
E\left(F_{2}, 2^{F_{2}}\right) \leq_{B} E\left(F_{2}, 9^{F_{3}}\right)\lceil A,
$$

which is enough, since $E\left(F_{2}, 2^{F_{2}}\right)$ is universal (see, e.g., Dougherty-Jackson-Kechris [DJK]).

LEMMA 4.3. There is a Borel injection $f: 2^{F_{2}} \rightarrow 9^{F_{3}}$ with $f\left(2^{F_{2}}\right) \subseteq A$ which preserves the group action of $F_{2}$ (i.e., for all $g \in F_{2}$ and $x \in 2^{F_{2}}, f(g \cdot x)=g \cdot f(x)$ ). So in particular

$$
E\left(F_{2}, 2^{F_{2}}\right) \leq E\left(F_{2}, 9^{F_{3}}\right)\lceil A .
$$


To prove this lemma, we will need the following technical sublemma.

Sublemma. For each $w \in F_{2} \backslash\{1\}$, there is a Borel injection $f_{w}: 2^{F_{2}} \rightarrow 6^{F_{2}}$ which preserves the group action of $F_{2}$ and satisfies

$$
f_{w}(x)(g)=f_{w}(x)(g w) \Longrightarrow g^{-1} \cdot x=w^{-1} g^{-1} \cdot x
$$

for all $g \in F_{2}$ and $x \in 2^{F_{2}}$.

We will assume this and complete the proof.

Proof of Lemma 4.3. Let $\left\{\alpha_{1}, \alpha_{2}\right\}$ be the generators of $F_{2}$ and $\left\{\alpha_{1}, \alpha_{2}, \alpha_{3}\right\}$ the generators of $F_{3}$. Define $f(x)$ for $x \in 2^{F_{2}}$ as follows:

(i) If $g \in F_{2}$, then $f(x)(g)=x(g)$.

(ii) If $g=h \alpha_{3}^{-p} g^{\prime}$, with $h \in F_{2}, p>0$, and $g^{\prime}$ not starting with $\alpha_{3}^{ \pm 1}$, then $f(x)(g)=2$.

(iii) If $g=h \alpha_{3}^{p} g^{\prime}$, with $h, g^{\prime}$ as in (ii) and $p>0, p \neq m_{j}^{(i)}$ for all $i, j$, then $f(x)(g)=2$.

(iv) If $g=h \alpha_{3}^{m_{j}^{(i)}} g^{\prime}$, with $h, g^{\prime}$ as in (ii), then $f(x)(g)=f_{w_{j}^{(i)}}(x)(h)+3$, where

$$
w_{j}^{(i)}=g_{n_{j}^{(i)}} g_{n_{j+1}^{(i)}}^{-1} .
$$

It is easy to check that $f$ is one-to-one and preserves the action of $F_{2}$. So it remains to verify that $f(x) \in A$.

So fix $i, j$ with

$$
\left(g_{n_{j}^{(i)}} \cdot f(x)\right) \mid B_{3}\left(1, m_{j}^{(i)}\right)=\left(g_{n_{j+1}^{(i)}} \cdot f(x)\right)\left\lceil B_{3}\left(1, m_{j}^{(i)}\right) .\right.
$$

If $d=\alpha_{3}^{m_{j}^{(i)}}$, then $d \in B_{3}\left(1, m_{j}^{(i)}\right)$, so

$$
f(x)\left(g_{n_{j}^{(i)}}^{-1} d\right)=f(x)\left(g_{n_{j+1}^{(i)}}^{-1} d\right)
$$

thus

$$
\begin{aligned}
f_{w_{j}^{(i)}}(x)\left(g_{n_{j}^{(i)}}^{-1}\right) & =f_{w_{j}^{(i)}}(x)\left(g_{n_{j+1}^{(i)}}^{-1}\right) \\
& =f_{w_{j}^{(i)}}(x)\left(g_{n_{j}^{(i)}}^{-1} w_{j}^{(i)}\right) .
\end{aligned}
$$

By the sublemma, $g_{n_{j}^{(i)}} \cdot x=\left(w_{j}^{(i)}\right)^{-1} g_{n_{j}^{(i)}} \cdot x=g_{n_{j+1}^{(i)}} \cdot x$, so

$$
\begin{aligned}
g_{n_{j}^{(i)}} \cdot f(x) & =f\left(g_{n_{j}^{(i)}} \cdot x\right) \\
& =f\left(g_{n_{j+1}^{(i)}} \cdot x\right) \\
& =g_{n_{j+1}^{(i)}} \cdot f(x) ;
\end{aligned}
$$

since $i, j$ were arbitrary, $f(x) \in A$.

It remains to prove the sublemma.

Proof of Sublemma. View $F_{2}$ as a rooted tree in the usual way ( 1 is the root of this tree, and there is an edge between $g$ and $g \alpha_{i}$ for any group element $g$ and generator $\alpha_{i}$ ). Thus $x \in 2^{F_{2}}$ is a labeling of this tree using labels 0,1 . Similarly 
for $6^{F_{2}}$. Then $g^{-1} \cdot x$ is the same labeling except that the root of the tree is at $g$ instead of 1 . So the condition

$$
\forall g\left[g^{-1} \cdot x \neq w^{-1} g^{-1} \cdot x \Longrightarrow f_{w}(x)(g) \neq f_{w}(x)(g w)\right]
$$

just means that if $x$, viewed from root $g$, is different from $x$ viewed from $g w$, then the label of $f_{w}(x)$ at $g$ is different from the label of $f_{w}(x)$ at $g w$. Moreover, to guarantee that $f_{w}\left(g^{\prime} \cdot x\right)=g^{\prime} \cdot f_{w}(x)$ for each $g^{\prime} \in F_{2}$, we will make sure that the value of $f_{w}(x)$ at any $g$ depends only on the labeling $x$ viewed from root $g$ (and not on $g$ itself).

Given $x \in 2^{F_{2}}$ and $g \in F_{2}$, we have two cases:

(I) $g^{-1} \cdot x=w^{-1} g^{-1} \cdot x$, i.e., $x$ looks the same from root $g$ and root $g w$ (note that this only depends on how $x$ looks from root $g$ ).

Then put $f_{w}(x)(g)=\langle x(g), 0\rangle$, where $\langle$,$\rangle is a bijection of 2 \times 3$ with 6 .

(II) $g^{-1} \cdot x \neq w^{-1} g^{-1} \cdot x$. So $x$ looks different from roots $g, g w$. In particular there is a least $n=n_{g}(x)$ so that for some $i, j \in \mathbb{Z}$ and $h \in F_{2}$ of length $n$ we have $x\left(g w^{i} h\right) \neq x\left(g w^{j} h\right)$. Clearly $n_{g w^{i}}(x)=n_{g}(x)$ for any integer $i$ (note that $\left(g w^{i}\right)^{-1} \cdot x \neq w^{-1}\left(g w^{i}\right)^{-1} \cdot x$ as well).

The functions $p_{j}: B_{2}\left(1, n_{g}(x)\right) \rightarrow 2$ given by

$$
p_{j}(h)=x\left(g w^{j} h\right)
$$

are thus not all equal. So fix $p \in 2^{B_{2}\left(1, n_{g}(x)\right)}$ with $Z=\left\{j \in \mathbb{Z}: p_{j}=p\right) \neq \varnothing$ and $p$ least such (in some ordering of $2^{B_{2}\left(1, n_{g}(x)\right)}$ fixed in advance). The value of $p$ would be the same if we started with $g w^{i}$ instead of $g$; the set $\tilde{Z}$ we would get from $g w^{i}$ is a translate of $Z(j \in \tilde{Z}$ iff $j+i \in Z)$.

Also $\left\{j \in \mathbb{Z}: p_{j} \neq p\right\} \neq \varnothing$. If $Z$ has a largest element $i_{0}$, let $f_{w}(x)(g)=$ $\langle x(g), 0\rangle$, if $i_{0}$ is even, and $f_{w}(x)(g)=\langle x(g), 1\rangle$, if $i_{0}$ is odd. If $Z$ has no largest element but has a least element $i_{0}$, define $f_{w}(x)(g)$ the same way. Proceed similarly if $\mathbb{Z} \backslash Z$ has a least or largest element. So assume both $Z$ and $\mathbb{Z} \backslash Z$ are unbounded in both directions. Put

$$
Z^{\prime}=\{j \in Z: j+1 \notin Z\} .
$$

Let finally $f_{w}(x)(g)=\langle x(g), 0\rangle$ if $0 \in Z^{\prime}, f_{w}(x)(g)=\langle x(g), 1\rangle$ if $0 \notin Z^{\prime}$, but the least positive element of $Z^{\prime}$ if odd, and $f_{w}(x)(g)=\langle x(g), 2\rangle$ if this least positive element is even.

This completes the definition of $f$; it is straightforward to verify that it has the desired properties.

This completes the proof of Theorem 4.1.

We conclude with another application of these ideas.

For a countable group $G$ consider the shift action of $G$ on $X^{G}$. We call $x \in X^{G}$ a left-free point if for all distinct $g, g^{\prime} \in G$ there exists $h \in G$ such that $x(h g) \neq$ $x\left(h g^{\prime}\right)$. We call $x \in X^{G}$ a right-free or just free point, if for all distinct $g, g^{\prime} \in G$ there exists $h \in G$ such that $x(g h) \neq x\left(g^{\prime} h\right)$; equivalently, $g \cdot x \neq g^{\prime} \cdot x$ for $g \neq g^{\prime}$, or simply $g \cdot x \neq x$ for all $g \neq 1_{G}$. Denote by $L F$ the set of left-free points and $F$ the set of free points. Note that $L F$ and $F$ are Borel $G$-invariant subsets of $X^{G}$. If $G$ is abelian, clearly $L F=F$. But $L F$ and $F$ are very different for free groups in the following sense.

THEOREM 4.4. The equivalence relation $E\left(F_{3}, 4^{F_{3}}\right)\lceil L F$ is universal for countable Borel equivalence relations but $E\left(F_{3}, 4^{F_{3}}\right)\lceil F$ is not. 
Proof. The equivalence relation $E\left(F_{3}, 4^{F_{3}}\right)\lceil F$ is not universal because it is treeable; see Kechris [K2]. For the first assertion we will show that $E\left(F_{2}, 2^{F_{2}}\right) \leq_{B}$ $E\left(F_{3}, 4^{F_{3}}\right)\lceil L F$.

Fix a left-free point $z_{0}$ in $\{2,3\}^{F_{2}}$. Define then $f: 2^{F_{2}} \rightarrow 4^{F_{3}}$ by:

(i) If $h \in F_{2}, f(x)(h)=x(h)$.

(ii) If $h \notin F_{2}$, express the reduced word for $h$ in the form $h=h_{1} \alpha_{3}^{ \pm 1} h^{\prime}$ with $h^{\prime} \in F_{2}$, and put $f(x)(h)=z_{0}\left(h^{\prime}\right)$.

It is easy to check that $x E\left(F_{2}, 2^{F_{2}}\right) y \Longleftrightarrow f(x) E\left(F_{3}, 4^{F_{3}}\right) f(y)$. It remains to verify that $f(x) \in L F$. Let $g$ and $g^{\prime}$ be distinct elements of $F_{3}$; we must find $h \in F_{3}$ such that $f(x)(h g) \neq f(x)\left(h g^{\prime}\right)$.

Consider two cases:

(1) $g^{-1} g^{\prime} \in F_{2}$. Then let $p \in F_{2}$ be such that $z_{0}(p) \neq z_{0}\left(p g^{-1} g^{\prime}\right)$, and let $h$ be such that $h g=\alpha_{3} p$. Then $f(x)(h g)=f(x)\left(\alpha_{3} p\right)=z_{0}(p) \neq z_{0}\left(p g^{-1} g^{\prime}\right)=$ $f(x)\left(h g g^{-1} g^{\prime}\right)=f(x)\left(h g^{\prime}\right)$.

(2) $g^{-1} g^{\prime} \notin F_{2}$. Let $h=g^{-1}$. Then

$$
f(x)(h g)=f(x)(1)=x(1) \in\{0,1\}
$$

but

$$
f(x)\left(h g^{\prime}\right)=f(x)\left(g^{-1} g^{\prime}\right)=z_{0}\left(h^{\prime}\right) \in\{2,3\}
$$

for some $h^{\prime} \in F_{2}$.

\section{References}

[A] S. Adams, An equivalence relation that is not freely generated, Proc. Amer. Math. Soc., $102(1988), 565-566$.

[AK] S. Adams and A. S. Kechris, Linear algebraic groups and descriptive set theory, preprint, 1999.

[ACH] A. Andretta, R. Camerlo, and G. Hjorth, Conjugacy equivalence relation on subgroups, preprint, 1999.

[DJK] R. Dougherty, S. Jackson, and A. S. Kechris, The structure of hyperfinite Borel equivalence relations, Trans. Amer. Math. Soc., 341 (1994), 193-225.

[DK] R. Dougherty and A. S. Kechris, The universality of recursive isomorphism on $\omega^{\omega}$, preprint, 1991.

[E] E. G. Effros, Transformation groups and $C^{*}$-algebras, Ann. of Math., 81(2) (1965), 38-55.

[FM] J. Feldman and C. C. Moore, Ergodic equivalence relations, cohomology and von Neumann algebras, I, Trans. Amer. Math. Soc., 234 (1977), 289-324.

[HKL] L. Harrington, A. S. Kechris, and A. Louveau, A Glimm-Effros dichotomy for Borel equivalence relations, J. Amer. Math. Soc., 3 (1990), 903-928.

[JKL] S. Jackson, A. S. Kechris, and A. Louveau, Countable Borel equivalence relations, in preparation.

[K1] A. S. Kechris, Amenable equivalence relations and Turing degrees, J. Symb. Logic, 56 (1991), 182-194.

[K2] A. S. Kechris, The structure of Borel equivalence relations in Polish spaces, Set Theory of the Continuum, H. Judah, W. Just, H. Woodin, Eds., MSRI Publications, Vol. 26, Springer-Verlag, New York, 1992, 89-102.

[K3] A. S. Kechris, New directions in descriptive set theory, Bull. Symb. Logic, 5 (1999), 161174 .

[KM] A. S. Kechris and Y.N. Moschovakis (Eds.), Cabal Seminar 76-77, Lecture Notes in Math., vol. 689, Springer-Verlag, 1978.

[N] M. G. Nadkarni, On the existence of a finite invariant measure, Proc. Indian Acad. Sci. Math. Sci., 100 (1990), 203-220.

[SS] T. Slaman and J. Steel, Definable functions on degrees, Cabal Seminar, 81-85, Lecture Notes in Math., vol. 1333, Springer-Verlag, 1988, 37-55. 
Department of Mathematics, Ohio State University, Columbus, Ohio 43210

E-mail address: rld@math.ohio-state.edu

Department of Mathematics, California Institute of Technology, Pasadena, CaliFORNIA 91125

E-mail address: kechris@caltech.edu 